Vol. 13, No 1-2 | 1er semestre 2019 - 2ème semestre 2019 Les industries culturelles à la conquête des plateformes?

\title{
Ulule, du courtage à la structuration des pratiques de création dans l'édition, la musique et l'audiovisuel
}

Manuel DUPUY-SALLE et Laurie SCHMITT

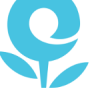

\section{OpenEdition}

Édition électronique

URL : http://journals.openedition.org/ticetsociete/3154

DOI : 10.4000/ticetsociete.3154

Éditeur

Association ARTIC

Édition imprimée

Pagination : 97-132

Référence électronique

Manuel DUPUY-SALLE et Laurie SCHMITT, « Ulule, du courtage à la structuration des pratiques de

création dans l'édition, la musique et l'audiovisuel », tic\&société [En ligne], Vol. 13, N 1-2 | 1er semestre 2019 - 2ème semestre 2019, mis en ligne le 20 avril 2019, consulté le 15 mai 2019. URL : http://

journals.openedition.org/ticetsociete/3154; DOI : 10.4000/ticetsociete.3154 
tic\&société - 13(1-2), 2019

\title{
Ulule, du courtage à la structuration des pratiques de création dans l'édition, la musique et l'audiovisuel
}

\begin{abstract}
Manuel DUPUY-SALLE
Manuel Dupuy-Salle est maître de conférences en sciences de l'information et de la communication à l'université Lyon 2. Chercheur à ELICO, ses travaux portent sur les mutations des pratiques culturelles et des formes de l'amateurisme contemporain au regard des usages sociaux des TIC numériques et de l'Internet. II s'intéresse également aux stratégies socioéconomiques des acteurs du Web collaboratif (crowdfunding, streaming, réseaux socionumériques, etc.) dans le cadre des mutations des industries culturelles, de l'information et de la communication. Manuel.DupuySalle@univ-lyon2.fr
\end{abstract}

\section{Laurie SCHMITT}

Laurie Schmitt est maître de conférences en sciences de l'information et de la communication à l'université Grenoble Alpes. Elle est chercheure au Gresec (Groupe de recherche sur les enjeux de la communication). Ses thématiques de recherche portent sur les mutations des industries culturelles et médiatiques, les usages de la photographie dans les médias et les stratégies d'acteurs accompagnant l'émergence de plateformes numériques et le développement de dispositifs médiatiques tels que le webdocumentaire ou le transmédia. Elle est l'auteure de l'ouvrage Quand les médias utilisent les photographies des amateurs (coédition INA-Bord de l'Eau, 2012). Elle est également responsable du Master InformationCommunication, parcours audiovisuel et médias numériques de l'université Grenoble Alpes. Laurie.Schmitt@univ-grenoblealpes.fr 
Ulule, du courtage à la structuration des pratiques de création dans l'édition, la musique et l'audiovisuel

\title{
Ulule, du courtage à la structuration des pratiques de création dans l'édition, la musique et l'audiovisuel
}

Résumé : Cet article s'intéresse aux plateformes de financement participatif en tant que dispositifs sociotechniques qui organisent la mise en relation entre différents acteurs des industries culturelles. Ces plateformes, en tant qu' intermédiaires » supplémentaires, participent à la structuration des pratiques de création dans le champ culturel, pour les acteurs de contenus qui s'en saisissent. Nous verrons ainsi comment le recours au crowdfunding par des acteurs des industries culturelles s'insère dans des tendances continues au sein des filières. Ensuite, nous envisagerons les mutations qui a contrario le sous-tendent, le financement participatif combinant pratiques anciennes et nouvelles. Enfin, nous traiterons des mobilisations différenciées du financement participatif selon les filières, les porteurs ou encore les plateformes. Notre réflexion prend appui sur l'analyse de 472 projets déposés sur la plateforme Ulule entre 2016 et 2017 et de dix entretiens semi-directifs menés auprès de porteurs de projets et du directeur général d'Ulule.

Mots-clés: crowdfunding, industries culturelles, courtage, intermédiation, création.

\begin{abstract}
This article focuses on crowdfunding platforms as socio-technical devices that organize connections between different actors in the cultural industries. As additional "intermediaries", these platforms help structure creative practices in the cultural field, for the actors of content that capture them. We examine how the use of crowdfunding by cultural industry players is part of an ongoing trend within various sectors. We then consider the mutations that underlie this phenomenon: crowdfunding combines old and new practices. Finally, we discuss how crowdfunding is mobilized differently according to sectors, carriers or platforms. Our study is based on the analysis of 472 projects on the Ulule platform between 2016 and 2017 and ten semi-structured interviews with project sponsors and the CEO of Ulule.
\end{abstract}


Keywords: crowdfunding, cultural industries, brokerage, intermediation, creation.

Resumen: Este texto se centra en las plataformas de crowdfunding, consideradas como dispositivos sociotécnicos que organizan la conexión entre los diferentes actores de las industrias culturales. Estas plataformas, en tanto que "intermediarios" suplementarios, participan en la estructuración de prácticas creativas en el campo cultural, y por tanto en los agentes que las utilizan. Se muestra que el uso del crowdfunding por parte de los actores de las industrias culturales se inscribe dentro de los cambios que tiene lugar en el seno de las hileras. Posteriormente, se analizan dichas mutaciones: el crowdfunding combina prácticas antiguas y nuevas. Finalmente, se muestran las diferentes movilizaciones diferenciadas de crowdfunding según sectores, actores o plataformas. El análisis se basa en el estudio de 472 proyectos presentados en la plataforma Ulule entre 2016 y 2017 y en diez entrevistas semiestructuradas con portadores de proyectos y director general de Ulule.

Palabras claves: crowdfunding, industrias culturales, corretaje, intermediación, creación. 
Ulule, du courtage à la structuration des pratiques de création dans l'édition, la musique et l'audiovisuel

\section{Les plateformes de crowdfunding au regard des industries culturelles}

L'objectif de cet article (mené dans le cadre du programme MODIPIC ${ }^{1}$ ) est d'interroger la place et les incidences des plateformes de crowdfunding (ici Ulule) dans le fonctionnement des industries culturelles et, plus précisément, dans les mutations et les reconfigurations des pratiques socioprofessionnelles de création qui s'y déploient.

Nous nous inscrivons dans le cadre de l'économie politique de la communication et, plus particulièrement, dans l'approche des industries culturelles (Huet et al., 1978 ; Mœglin, 2012 ; Miège, 2017). Celle-ci cherche à saisir, selon une posture critique, historiciste et socioéconomique (Guibert, Rebillard et Rochelandet, 2016), les caractéristiques des différentes filières culturelles (cinéma et audiovisuel, édition, musique enregistrée, presse, jeux vidéo, infomédiation) et les modalités types par lesquelles les contenus y sont produits, diffusés et consommés. Cette approche a pour particularité de saisir les logiques à la fois sociales, professionnelles et financières en jeu dans l'organisation des chaînes de coopération entre acteurs. Elle permet in fine d'éclairer le renouvellement des rapports de force entre les acteurs émergents et les acteurs traditionnels ainsi que les mutations et les enjeux de ces changements sur les modes d'existence des contenus culturels.

Depuis un certain nombre d'années, les acteurs traditionnels des industries culturelles sont confrontés à la nécessité de composer et de négocier leurs stratégies avec de nouveaux acteurs, parfois puissants, se positionnant sur les contenus culturels: les industries de la communication et, notamment, celles du Web (Bouquillion, 2008; Bouquillion, Miège et Mœglin, 2013). Ainsi, à l'instar des acteurs du « Web collaboratif »(Bouquillion et Matthews, 2010), des plateformes

\footnotetext{
${ }^{1}$ Le programme MODIPIC (Mobilisation différenciée des plateformes par les industries culturelles: de la production à la diffusion des contenus) fait partie du projet structurant PLATEFORMES du Labex ICCA (Industries culturelles et création artistique) et a pour objectif de comprendre comment les industries culturelles se positionnent par rapport aux plateformes nationales collaboratives (Dailymotion et Ulule).
} 
de streaming (Perticoz, 2012) ou d'hébergement vidéo (Bullich, 2015), les acteurs du crowdfunding en ligne peuvent aussi être considérés comme de nouveaux entrants confrontés aux filières culturelles. Sont-ils en capacité, comme le prétendent la plupart des discours d'escorte empreints d'idéologie "Web 2.0 » (Bénistant et Marty, 2016), de renouveler, voire de remplacer, les traditionnelles voies de production des contenus culturels ? Si des travaux attestent, avec prudence, que ces plateformes facilitent le développement de certaines formes d'autoproduction (Onnée et Renault, 2013 ; Bubendorff, 2014 ; Creton et Kitsopanidou, 2016 ; Rallet et Rochelandet, 2016), ces acteurs contribuent aussi à un renforcement des mécanismes classiques d'industrialisation et de marchandisation dans les filières culturelles (Matthews, Rouzé et Vachet, 2014 ; Rouzé et Matthews, 2018). En effet, les incidences de ces " plateformes d'intermédiation " (Matthews, 2015) sur l'organisation des filières se caractérisent par leur capacité à insérer, dans des processus plus rationalisés, certaines pratiques et acteurs culturels jusqu'ici laissés en marge des filières (certaines formes d'amateurisme, par exemple), à élargir les formes du travail culturel sous les traits du digital labor (Rouzé et Matthews, 2018) ou encore à contribuer à la structuration de filières culturelles jusqu'ici peu industrialisées (Matthews, Rouzé et Vachet, 2014).

Dans la continuité de ces pistes de recherche, notre hypothèse est que ces plateformes, à travers leur fonctionnement et leurs sollicitations, participent à une actualisation, voire à une restructuration, des pratiques de création. Pour vérifier ceci, nous partons des façons dont les acteurs traditionnels se saisissent des plateformes pour développer leur projet. Cette entrée permet d'éclairer les conditions, les perspectives, les acteurs et les types de contenus dans lequel le recours au crowdfunding s'envisage. L'objectif est de saisir les logiques d'appropriation des plateformes qui sont à l'œuvre dans différentes filières, de regarder les convergences et les différences d'usages, mais aussi d'identifier dans quelle mesure les plateformes centralisent et organisent des logiques de fonctionnement interfilière, en rapprochant des acteurs qui n'ont pas l'habitude d'être mis en contact ni de travailler ensemble. 
Ulule, du courtage à la structuration des pratiques de création dans l'édition, la musique et l'audiovisuel

Notre analyse s'appuie sur le cas d'Ulule ${ }^{2}$. Start-up française fondée en 2009, elle crée Ulule.com en 2010 et se positionne en 2019 comme la plateforme leader en France sur le secteur culturel en ce qui concerne les montants collectés (devant KissKissBankBank et Kickstarter). Elle se définit sur son site comme " une opportunité de financement de projets créatifs, innovants ou solidaires ". Généraliste, elle ne se spécialise sur aucune catégorie particulière de contenus culturels ${ }^{3}$. Sur le plan économique et à l'instar de ses concurrentes, elle fonctionne selon un modèle " de don " (et non de prêt ou d'investissement), où les contributeurs participent au financement des projets en échange de contreparties non financières. La plateforme se rémunère en récupérant de $5 \%$ à $8 \%$ du montant collecté sur toute campagne financée avec succès. Si celle-ci échoue (au sens où elle n'atteint pas le montant demandé), les dons sont rendus aux contributeurs.

La sélection de cette plateforme s'explique d'abord par le constat qu'il n'existe en France aucune étude approfondie de cet acteur majeur du crowdfunding culturel, pourtant régulièrement sollicité par les industries culturelles et sur lequel s'y déploient de nombreux projets. Ensuite, il se justifie par le positionnement généraliste de la plateforme qui, en se plaçant au carrefour de plusieurs filières, lui permet d'entrer en relation avec des acteurs traditionnels variés, tant sur le plan des métiers (producteurs de films, éditeurs, musiciens) que des statuts (étudiants, professionnels, semi-pro).

L'enquête s'appuie en premier lieu sur une analyse de contenu appliquée à 472 projets (issus des catégories d'Ulule « film-vidéo », " édition-journal » et " musique ») déposés sur la plateforme entre 2016 et 2017 . Notre échantillon est double. II concerne pour partie des projets en cours de financement pendant le temps de l'enquête. Ainsi, 322 projets ont été analysés, issus de trois coups de sondes réalisés entre avril 2016 et février 2017. Ce choix s'explique par la volonté d'avoir un panorama de projets déposés " au quotidien », sur une période définie et sans présumer de leur statut, succès ou échec futur. II favorise justement, a posteriori, l'identification les

\footnotetext{
${ }^{2}$ Cette étude a été menée en collaboration avec Dominique Cartellier (GRESEC, Université Grenoble Alpes).

${ }^{3}$ Elle déclare avoir financé, en 2018, plus de 23120 projets (avec $65 \%$ de taux de réussite) et avoir atteint 110815186 euros collectés au $1^{\mathrm{er}}$ juin 2018.
} 
cas d'échecs éventuels et permet de les prendre en compte dans l'analyse, ces échecs disparaissant de la plateforme une fois leur campagne terminée ${ }^{4}$. L'autre partie de l'échantillon concerne les projets financés et triés par montant collecté, soit 50 projets pour chaque rubrique susmentionnée. La constitution de ce nouvel ensemble a pour objectif d'identifier les caractéristiques des projets à succès (statut des porteurs, liens éventuels avec l'industrie traditionnelle) et de comparer leur différence avec les autres projets. Pour ces deux échantillons, l'analyse de contenu s'est effectuée sur les pages des projets à travers une collecte d'items variés: les caractéristiques ${ }^{5}$, les acteurs $^{6}$, la mise en valeur du projet ${ }^{7}$, le financement ${ }^{8}$ et la campagne de crowdfunding ${ }^{9}$. Ces données ont été traitées par une analyse statistique visant à dégager certaines tendances. Elles ont aussi donné lieu à un travail d'analyse qualitative considérant en détail, si besoin, les spécificités de tel ou tel projet. L'étude n'est ni exhaustive ni généralisable, mais elle favorise l'identification de logiques autour du type de projets, des acteurs et des industries culturelles les plus concernées par ces modes de financement.

L'enquête s'appuie en second lieu sur dix entretiens semidirectifs permettant d'appréhender les pratiques, les stratégies et les représentations des acteurs concernés. Une diversité d'interrogés a été privilégiée : d'un côté, le directeur général d'Ulule, afin d'avoir des éléments sur les orientations et les stratégies de la plateforme et, de l'autre, des porteurs sélectionnés à partir de caractéristiques clés, à savoir le statut professionnel ou les origines, les démarches et les finalités du

\footnotetext{
${ }^{4}$ Les projets ne collectant pas les sommes demandées sont archivés dans la base de données d'Ulule, mais ne sont pas accessibles à partir du moteur de recherche de la plateforme.

${ }^{5}$ Ont été référencés : le titre, le descriptif, le type, le genre de projet et le public visé.

${ }^{6}$ Nous avons identifié : le porteur, le nombre de projets créés et soutenus par le porteur, la localisation du projet (par ville et par région), le bénéficiaire de la collecte, les partenaires du projet, les Official Users.

${ }^{7}$ Nous nous sommes intéressés aux rubriques de référencement du projet et au nombre de news publiées par les porteurs.

${ }^{8}$ Nous avons référencé : les phases de production auxquelles iront le financement demandé (conception-production, postproduction, distribution, campagne de crowdfunding), le type d'activités renvoyant à ces phases (enregistrement, mixage, mastering, pressage), le nombre de contributeurs, le montant demandé, la somme collectée et le résultat final de la campagne (projet financé ou non).

${ }^{9}$ Nous avons collecté : le nombre de paliers, le type de contreparties, les montants minimum et maximum des contreparties.
} 
Ulule, du courtage à la structuration des pratiques de création dans l'édition, la musique et l'audiovisuel

projet. Les entretiens ont été conduits autour de ces thèmes. Ces acteurs sont : trois réalisateurs, un documentariste télé, un journaliste, un éditeur de magazine, deux éditeurs d'ouvrage et un directeur de label indépendant de musique.

Cet article se structure en trois temps. II envisage d'abord comment le recours au crowdfunding s'insère dans la socioéconomie des filières. Ensuite, il observe à quelles mutations la plateforme contribue et, enfin, traite des mobilisations différenciées du service par les acteurs culturels.

\section{Des tendances continues au sein des filières}

Ulule bouleverse-t-elle les processus classiques de production des industries culturelles? Notre analyse montre qu'elle s'intègre plutôt dans l'existant et qu'elle reproduit des traits constitutifs de ces dernières.

\subsection{Une polarisation des projets}

L'analyse des projets déposés sur Ulule rend compte d'un renforcement du mouvement de polarisation présent au sein des filières, entre un petit nombre de blockbusters et un grand nombre de productions de niche fonctionnant à la limite de la rentabilité (Bouquillion, 2008, p. 280).

D'un côté, les projets rapportant le plus de financement (de type blockbusters, entendu ici dans le succès de la récolte) sont peu nombreux et portés, pour la majorité, par des maisons de production et d'édition : "Noob le film » (Olydri Studio), « Hero Corp Saison 5 » (Calt Production), "Stupeflip Nouvel Album » (ETIC System) ou encore "soutenez Nice Matin ». Les projets audiovisuels financés ayant récolté le plus d'argent sont des documentaires $(34 \%)$ ou des longs métrages fictionnels $(14 \%)$. Pour les projets de la rubrique " édition et journal », il s'agit de magazines (24\%) ou de médias en ligne (20\%). Parmi les projets musicaux, les albums sont les plus représentés $(40 \%)$

De l'autre côté, les projets de niches prolifèrent. Ce sont des productions aux moyens contraints, souvent portés par des individus et non des sociétés. Ils relèvent pour l'essentiel dans les projets audiovisuels: de courts métrages $(50 \%)$; les projets éditoriaux: de livres ( $89 \%)$; et les projets musicaux : 
d'albums (60\%), d'EP (18\%) ou de clips vidéo (15\%).

Ces catégories identifiées semblent témoigner d'une faible incitation à la création de contenus originaux (Bouquillion, Miège et Mœglin, 2013). En effet, les projets audiovisuels « innovants » sont minoritaires : un projet multisupport $(3 \times 3$, un thriller fantastique) et trois projets de plateforme (telle Tënk). Les projets déposés se calquent bien souvent sur le type, le genre, le format des productions traditionnelles du marché. Parmi les projets musicaux, $53 \%$ sont des albums et $26 \%$ relèvent du genre musical «rock». Les projets proposés apparaissent également fédérateurs, dans le sens où ils réunissent des communautés. À ce titre, les projets de livres en cours de financement (Figure 1) relèvent de la littérature de genre (science fiction, à hauteur de $12 \%$ ), de beaux livres/livres illustrés (11\%), ou encore de livres touristiques ou de voyage $(13 \%)$. 
Ulule, du courtage à la structuration des pratiques de création dans l'édition, la musique et l'audiovisuel

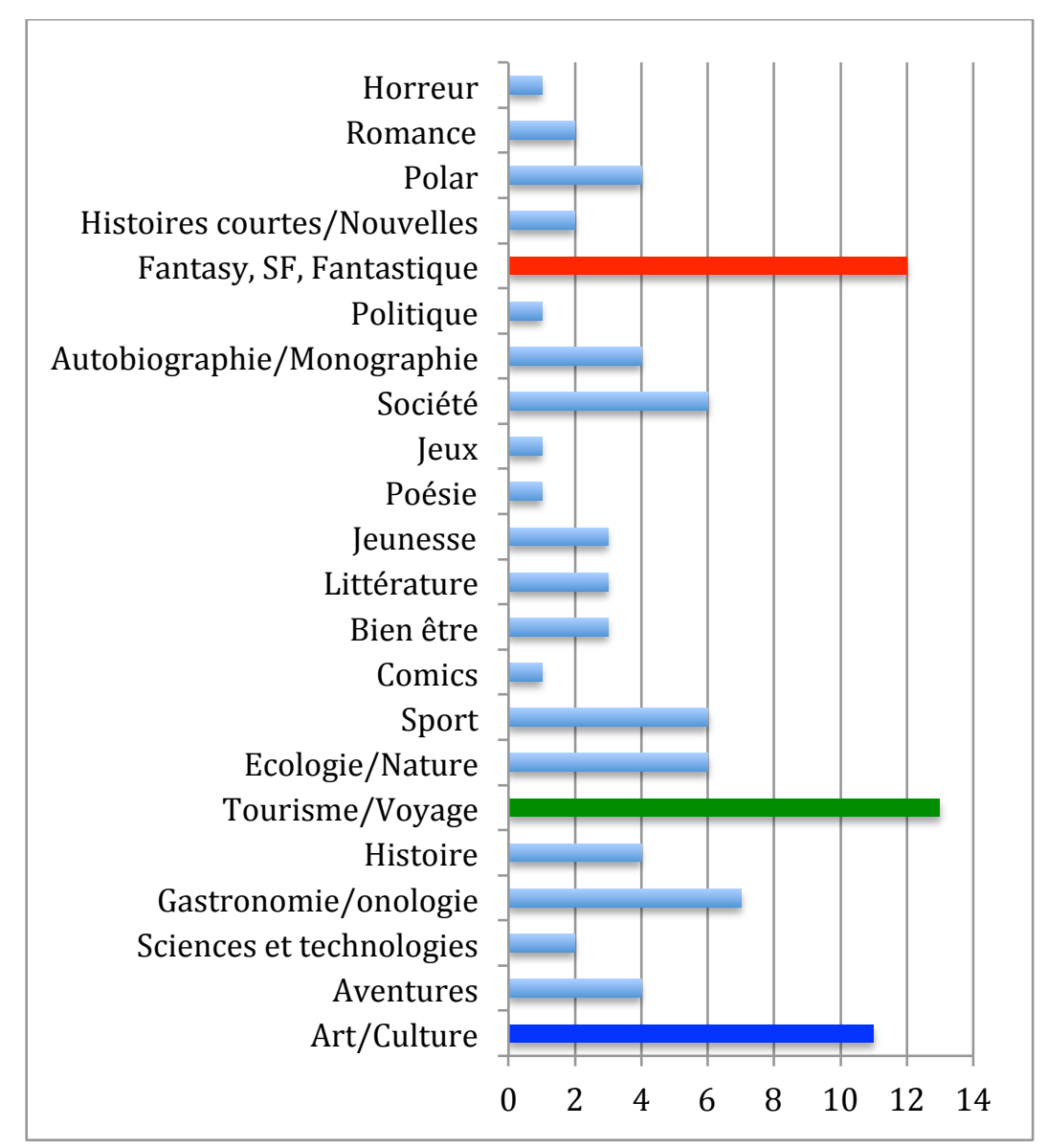

Figure 1. Genres déclarés pour les projets éditoriaux

\subsection{Une volonté de réduire l'incertitude}

Les industries culturelles connaissent une constante imprévisibilité du marché (Bouquillion, Miège et Mœglin, 2013 ; Miège, 2017). Face à cet aléa, elles développent des stratégies de réduction de l'incertitude : I'utilisation de sondages d'opinion et de techniques du marketing, l'appel au star system (Chantepie et Le Diberder, 2005), le développement d'émissions pilotes ou la reproduction de formules déjà éprouvées. Le recours aux plateformes de crowdfunding, envisagé par les acteurs culturels comme des laboratoires de test, grandeur nature, de projets et de porteurs est l'une de ces stratégies (Benghozi, 2013 ; Dupuy-Salle, 2016). Comment se caractérise-t-elle sur Ulule? 
En amont (préproduction), le crowdfunding offre un moyen, pour des projets audiovisuels, de s' « amorcer " (Matthews, Rouzé et Vachet, 2014, p. 9) en facilitant le montage d'un dossier de préfinancement de production. II se présente comme la réponse à un système de production contraint et difficile d'accès: "Les projets nouveaux médias sont de moins en moins soutenus par les distributeurs. L'avantage des plateformes de crowdfunding est de pouvoir fonder une communauté qui diffuse le projet et qui remplace presque les distributeurs », précise une journaliste interrogée. Ulule est également perçue, par les porteurs, comme un système de garantie, de certification, de viabilité du projet, voire d'une " prise de risque minimum ", indique un éditeur de livres. La campagne de financement participatif est également vue comme un moyen de "rentrer dans ses frais " et de se rembourser en couvrant, comme l'indiquent deux réalisateurs rencontrés, les dépenses d'un tournage déjà effectué et financé sur fonds propres. Enfin, à l'échelle de producteurs ou d'éditeurs, le crowdfunding représente une stratégie de gestion de l'incertitude, par une veille régulière, favorisant la découverte de nouveaux " talents ". Ulule offre aussi la possibilité de tester des projets et des créations auprès de publics pour y déceler les signes d'un intérêt, voire d'un succès futur.

En aval, le recours au crowdfunding apparaît comme une modalité de maîtrise plus aisée de la diffusion d'un contenu. II se dessine comme une occasion de commercialiser un produit ou d'en relancer une édition (en modèle de prévente). II s'agit, par exemple, de revaloriser un projet du catalogue peu exploité et peu rentable en créant, à l'aide de la campagne, des éditions " collector». Un producteur de label musical interrogé mentionne à ce titre le succès de la réédition, en vinyle, d'un album paru à la fin des années 1990 ayant subitement suscité, sur YouTube, un engouement renouvelé de la part d'un public de fans.

Mais au-delà de ces opportunités, le financement participatif reste perçu comme un moyen, qui parmi tant d'autres (parrainage, mécénat), demeure limité pour faire face aux difficultés classiques de financement des projets: "Avoir recours à une plateforme de crowdfunding, c'est juste dans une logique de survie! On récupère les miettes. On ne vit pas correctement du crowdfunding. Le crowdfunding, c'est faire 
Ulule, du courtage à la structuration des pratiques de création dans l'édition, la musique et l'audiovisuel

avec des bouts de ficelles! », souligne un documentariste. En somme, l'utilisation d'Ulule est une illustration supplémentaire de la prégnance de l'incertitude dans les industries culturelles et de son intégration, voire de son intériorisation, dans les stratégies professionnelles et artistiques (Miège, 2017).

\subsection{Un vivier de nouveaux talents}

Au sein des industries culturelles, le recours à des viviers est ancré et structurel.

Ce réservoir de main-d'œuvre artistique est composé de trois viviers [...] : le vivier constitué de jeunes talents que les éditeurs ou maisons de production testent; le vivier formé de l'ensemble des professionnels (mal rémunérés ou en situation de chômage chronique [...]) ; et le vivier composé de tous les non-professionnels (artistes en formation, amateurs, semi-professionnels, formateurs) [...]. (Miège, 2017, p. 35-36.)

Les acteurs présents sur Ulule correspondent majoritairement au vivier amateur (le troisième), suivi du deuxième et, dans une moindre mesure, du premier. L'analyse du type d'acteurs présents sur Ulule (porteurs de projets et Official Users) témoigne de deux positionnements stratégiques.

Du côté des porteurs de projets, la plateforme est investie comme une aide à l'organisation et à la structuration du travail de création, tel un tremplin d'entrée dans le secteur. Les industries culturelles accordent une importance au travail de création en voulant le préserver. Dans ce contexte, l'organisation du travail de conception conserve des marques fortes de la production artisanale, tandis que celui de production, et surtout de distribution, répond à des logiques industrielles. Quand elle est sollicitée pour intervenir en amont des projets, Ulule ne remet pas en question cette organisation. Bien au contraire, elle la reproduit et l'allonge. Ainsi, elle rassemble souvent des individus, des particuliers ou encore des petits acteurs industriels (des franges) souhaitant créer, par plaisir ou par quête de professionnalisation.

Les statuts des porteurs de projets déclarés par Ulule sur son site s'organisent comme suit : des particuliers (50\%), des associations $(35 \%)$ et des entreprises $(15 \%)$, soit un ratio constitué à $50 \%$ de personnes physiques et à $50 \%$ de 
personnes morales. Nos résultats sont sensiblement différents si l'on distingue les projets en cours de financement de ceux financés et triés par montant récolté. Pour les projets en cours de financement entre 2016 et 2017, les personnes physiques sont majoritaires et représentent $82 \%$ des projets musicaux, $72,73 \%$ des projets éditoriaux et $70,49 \%$ des projets audiovisuels. Ces chiffres sont inversés sur les projets financés et triés par montant récolté, où la présence de personnes morales se situe à hauteur de $78 \%$ pour les projets éditoriaux, $64 \%$ pour les projets audiovisuels et $38,78 \%$ pour les projets musicaux. Les porteurs ont souvent des statuts précaires (par ex., intermittents du spectacle) et cumulent, pour ceux rencontrés, les activités: un autoentrepreneur dans la production audiovisuelle, un journaliste et chef de projets ; un chargé de communication, directeur " en dilettante " d'un label de musique; un documentariste télé, fondateur d'une société de production audiovisuelle et créateur d'un média en ligne.

Les variations observées entre projets et filières s'expliquent par la structuration même de celles-ci. Ainsi certaines sont-elles plus organisées, telle la filière audiovisuelle. Les sociétés de production se tournent souvent vers les institutions publiques, tel le CNC (Centre national de la cinématographie et de l'image animée). Parmi les projets analysés, les sociétés de production audiovisuelle représentent seulement $6 \%$ des porteurs de projets en cours de financement, contre $24 \%$ dans ceux triés par montant récolté. Dans les deux cas, les réalisateurs sont davantage mis en avant comme porteurs de projets et les sociétés de production apparaissent plutôt comme partenaires : BlackRose Production, Filmeek, Jolis Mômes Production, Pur Prod', Jayan films, Les Films de la Falaise, etc. Ces sociétés sont des "franges » et non des « majors » du secteur. Dans la musique, les labels représentent $2 \%$ des porteurs pour les projets en cours de financement et $4 \%$ pour ceux financés et triés par montant récolté. Les labels référencés sont tous de petits indépendants (MusTraDem, Fakir music, InFiné). À l'inverse, les maisons d'édition représentent $15 \%$ des porteurs pour les projets en cours de financement et $12 \%$ pour ceux triés par montant collecté. Les éditeurs présents sont bien souvent spécialisés dans la littérature de genre et n'ont pas attendu les plateformes de crowdfunding pour faire appel à leurs communautés de lecteurs (Mnémos, Actusf, Les moutons électriques, Le poisson soluble, Balat, Le vent se lève). 
Ulule, du courtage à la structuration des pratiques de création dans l'édition, la musique et l'audiovisuel

Certains éditeurs rencontrés connaissant bien le système de production se sont appuyés, dans leur campagne de crowdfunding, sur chapitre.com et France Loisir pour préacheter le livre - les deux distributeurs renvoyant alors à la plateforme Ulule.

Les Official Users (partenaires d'Ulule) sont a priori mobilisés sur des projets (en apparaissant comme une sorte de label) pour aider les plus " prometteurs » à sortir du lot et à être plus visibles. En retour, leur bénéfice est « d'avoir une visibilité sur la collecte et un positionnement innovant » (ulule.com), soit d'obtenir, eux aussi, des retombées favorables en matière de communication. Les Official Users sont des acteurs qui investissent, financièrement ou symboliquement, sur des projets déposés : "C'est un statut de membre spécial, réservé aux structures officielles (médias, fondations ou entreprises). Les particuliers ne peuvent pas devenir Official User " (rubrique " $F A Q$ »). Dans les projets étudiés, ceux présents ne sont pas exclusivement issus des industries culturelles. Loin de là, les acteurs sont divers: des banques (BNP Paribas), des assurances (MAIF), mais aussi des acteurs du Web (Dailymotion). Le partenariat avec Dailymotion ${ }^{10}$ repose sur un principe d'abondement: l'entreprise double les dons des contributeurs, dans une certaine limite. Notons qu'il revient aux porteurs de démarcher et de contacter les Official Users qui pourraient potentiellement soutenir le projet et de prévoir des contreparties pour l'entreprise partenaire en cas d'abondement.

Les "dispositifs d'intégration des partenaires ", pour reprendre les propos d'Ulule, sont divers. À l'abondement sur une collecte s'ajoutent des appels à projets lancés par les Official Users, des jeux-concours, mais également du repérage de nouveaux talents (méthodes de sourcing) développé, par exemple, par Hello Play!, une filiale d'Hello Bank, banque mobile du groupe BNP Paribas. Parfois, les Official Users présents viennent en appui pour leurs compétences en matière de création de campagnes de financement participatif (La Boîte militante), de connaissance du secteur (TV5 Monde, jouant ainsi un rôle de reconnaissance symbolique), d'activités de

\footnotetext{
${ }^{10}$ Dailymotion est présent sur Ulule depuis 2012. II a à son actif 73 projets soutenus et 40 projets suivis, ce qui se traduit par: "Ce projet participe au programme Dailymotion » ou encore « Dailymotion soutient très fort ce projet. Pour chaque euro versé par un internaute, Dailymotion versera $1 €$ (dans la limite de $4000 €)$. "
} 
promotion (Les Suricates ou Wiseband) ou encore de soutien financier (BNP Paribas, dans la limite de 10000 euros). Dès lors, ces Official Users allouent des services aux porteurs de projets. Hello Play ! se présente d'ailleurs comme un service de crowdfunding dédié à la musique.

Par ces opérations de partenariat entre Ulule et des Official Users aux statuts et aux origines sectorielles divers, la plateforme orchestre des mises en contact relativement inédites entre des acteurs extérieurs aux industries culturelles (des banques, des marques de grande consommation) et des porteurs de projets (souvent amateurs). Cette activité d'entremise peut avoir, sur le long terme et ce qui reste à vérifier en détail, des effets d'acculturation réciproques, notamment sur le plan des discours, des valeurs et des normes de travail. Or si Ulule peut de cette façon contribuer à « faire parler un langage commun " à ces différents partenaires (Matthews, Rouzé et Vachet, 2014, p. 30), les porteurs de projets interrogés n'identifient pas clairement le programme Official Users et ne sont ni conscients des soutiens ni de leurs enjeux, alors même que des Official Users sont affichés sur leur page de projet. Finalement, ces partenariats ont une incidence toute relative sur les projets dès lors qu'ils n'apportent, a posteriori, aucune aide ni sur le financement ni sur la diffusionvalorisation-promotion du projet une fois la campagne de collecte terminée.

Si Ulule remplit donc des fonctions de courtage entre acteurs hétérogènes (culturel et hors culturel), elle n'organise pas d'elle-même de rapprochements directs entre les acteurs culturels, ni au sein d'une même filière (réalisateurs et producteurs, par exemple) ni au sein de filières variées (entre réalisateurs et musiciens, par exemple). Si des liens se tissent, ils se font indirectement par des stratégies spécifiques aux porteurs, non par un dispositif pensé et impulsé par la plateforme.

En définitive, Ulule ne déstructure pas les caractéristiques socioéconomiques classiques des industries culturelles (Miège, 2017). Au contraire, non seulement son fonctionnement retraduit les mêmes "règles du jeu " (Mœglin, 2007), mais la plateforme contribue à en accentuer certains aspects. Ainsi en est-il de la logique de "polarisation " des contenus qui se retrouve pleinement sur la plateforme, mais avec une 
Ulule, du courtage à la structuration des pratiques de création dans l'édition, la musique et l'audiovisuel

particularité, celle de la démultiplication de projets " amateurs » (au sens de non professionnels) qui restent d'ailleurs relativement peu innovants et originaux sur le plan d'une prétendue "diversité » artistico-esthétique. L'aléa de production-consommation (Mœglin, 2007) s'y retrouve aussi renouvelé.

Si Ulule permet, dans certains projets, de le réduire, sachant qu'une campagne réussie peut servir d'argument pour l'obtention de fonds supplémentaires, il n'en reste pas moins que la plateforme ajoute une étape supplémentaire d'incertitude à travers le procédé même de campagne de collecte, puisque sa réussite n'est jamais garantie d'avance. Enfin, ce sont les mêmes viviers classiques de travailleurs culturels qui apparaissent sur Ulule, néanmoins avec un élargissement considérable de la frange " amateur " (entrants, semi-pro, prépro), dont la réussite éventuelle de campagnes de financement n'aboutit pas systématiquement à des débouchés favorables pour les étapes de diffusion-consommation situées en aval.

\section{Des mutations plurielles au sein des filières}

La mobilisation d'Ulule s'articule à des mutations en cours au sein des filières. La plateforme s'y inscrit, mais vient aussi y apporter ses particularités. Lesquelles ?

\subsection{Un renforcement de l'intermédiation}

Le développement de plateformes de crowdfunding relève d'un phénomène de sophistication de l'intermédiation au sein des industries culturelles: " davantage qu'une déstructuration, se dessine plutôt une restructuration des modes de production et de consommation des biens culturels et médiatiques. De nouveaux acteurs profitent du déploiement du numérique pour s'intercaler entre créateurs et internautes " (Guibert, Rebillard et Rochelandet, 2016, p. 53). En effet, Ulule se présente comme un acteur supplémentaire de la chaîne de coopération d'acteurs dans des filières, ou plus spécifiquement des sousfilières peu structurées telles que la $\mathrm{BD}$, le clip vidéo ou la musique live. Ces constats rejoignent les propos de Matthews, Rouzé et Vachet (2014), qui rappellent que 
les plateformes de crowdfunding [...] contribuent à la réorganisation interne de filières jusque-là peu ou pas structurées industriellement. Ceci est particulièrement visible dans le cas de la musique live, où de nombreuses études [...] ont mis en avant la permanence d'un " écosystème " diversifié, marqué par un nombre significatif d'acteurs de taille limitée [...], fonctionnant sur des territoires plus ou moins restreints et avec des procédures hétérogènes et assez peu codifiées. (p. 170.)

En ce sens, Ulule active, dynamise ou organise les relations entre acteurs de différentes filières. Certains projets sont d'ailleurs intégrés dans de multiples catégories (musique, vidéo, édition, etc.).

Ulule intervient peu sur la production et la direction artistique et esthétique des contenus. Quand elle le fait, c'est dans la présélection qu'elle exerce en amont de leur mise en ligne. Des " critères d'éligibilité », imposés aux porteurs, ont ainsi été progressivement définis pour évaluer leur «potentiel de collecte »: la "portée collective " du contenu ou le " réalisme » du financement y sont mesurés. À ceci s'ajoutent plusieurs conditions nécessaires pour que le projet soit validé par l'équipe, c'est-à-dire avoir au moins cinq soutiens de départ ou élaborer a minima quatre contreparties (soit quatre échelles de montants).

Ulule se dessine moins comme un acteur directement concerné par les aspects créatifs des projets que comme un acteur dont l'expertise se rapproche de la gestion des transactions financières. D'ailleurs, les dirigeants de la plateforme ne sont pas issus de milieux culturels, mais du Web (Doctissimo) et d'un cabinet d'audit. Ulule se positionne en tant que "place de marché » de projets, en BtoB (Business to Business) en direction de ses partenaires et en BtoC (Business to Consumer) en direction des contributeurs. En 2016, les montants totaux des transactions effectuées s'élèvent à 25370299 euros. Le rachat en 2017 de KissKissBankBank, plateforme concurrente d'Ulule, par la Banque postale est révélateur de ce phénomène de congruence qu'entretient le crowdfunding avec la sphère financière. Le modèle d'affaires d'Ulule est, à l'instar des autres acteurs du crowdfunding, hybride et expérimental (Matthews, Rouzé et Vachet, 2014). II repose d'abord sur des commissions prélevées sur les sommes 
Ulule, du courtage à la structuration des pratiques de création dans l'édition, la musique et l'audiovisuel

collectées lors de la réussite d'un projet (entre $5 \%$ et $8 \%$ selon le volume de fonds collectés et du moyen de paiement utilisé : $\mathrm{CB}, \mathrm{PayPal}$, chèque), ce qui représente au total $60 \%$ du chiffre d'affaires, comme le précise le directeur général de la plateforme. Les $40 \%$ restant proviennent ensuite de partenariats (avec les Official Users) ou d'autres formes de commandite. En outre, Ulule a effectué en 2016 une collecte de fonds de 5 millions d'euros auprès de Citizen Capital, MAIF et BNP Paribas, lui permettant de diversifier son activité par la création de trois nouveaux services: Okpal, un service de collecte de fonds entre particuliers ; une boutique physique à Paris commercialisant divers projets réalisés grâce au site : et le site Kickoff, une " boîte à outils » à destination des créateurs et comprenant des appels à projets, des réductions chez des sociétés partenaires pour les utilisateurs d'Ulule et des médias prêts à soutenir une campagne de crowdfunding.

Ulule repose plus précisément sur une logique de plateforme (aux sens économique et commercial), répondant au modèle du courtage (Mœglin, 2007). En effet, Ulule organise les transactions, gère les paiements et prélève des commissions. Ainsi ses obligations sont-elles d'offrir une sécurisation des transactions et d'assurer une délivrance des fonds. En revanche, Ulule précise clairement (rubriques «CGU » et " $F A Q$ ») qu'elle n'est ni partie prenante au contrat conclu de fait entre un contributeur et un porteur de projet ni ne garantit les projets. Aucune vérification n'est faite par Ulule de l'utilisation de l'argent par les porteurs. L'équipe de la plateforme conseille sur le montant à collecter, le choix des contreparties, la répartition des coûts des contreparties et la durée de la campagne (en moyenne 45 jours). Un documentariste énonce en entretien: "Ulule nous conseille pour chiffrer non pas la somme dont nous avons besoin, mais la somme que nous pouvons a priori récolter ». La plateforme ne vient pas mettre en contact des artistes et des publics, comme cela est parfois annoncé. Elle ne prend pas non plus les responsabilités inhérentes au statut d'éditeur ou de producteur plus traditionnel. Si le décret $n^{\circ} 2001-609$ du 9 juillet 2001 (art. II-I-4) ${ }^{11}$ précise que le producteur « prend

\footnotetext{
${ }^{11}$ Relatif à la contribution des éditeurs de services de télévision diffusés en clair par voie hertzienne terrestre en mode analogique au développement de la production d'œuvres cinématographiques et audiovisuelles.
} 
personnellement ou partage solidairement l'initiative et la responsabilité financière, technique et artistique de la réalisation de l'œuvre et en garantit la bonne fin ", ce n'est pas le cas pour Ulule, qui laisse, sans solidarité toutes ces responsabilités aux porteurs. Plus précisément, ces derniers ont l'obligation de délivrer les contreparties promises, sans toutefois avoir l'obligation de réaliser le projet. En cas de manquement à ces obligations, leur responsabilité civile ou pénale peut être engagée, non celle d'Ulule.

\subsection{Une réactualisation du financement participatif}

Comme le soulignent Matthews, Rouzé et Vachet (2014) et Uricchio (2014), en citant les exemples de la construction du socle de la Statue de la Liberté ou encore le modèle de la souscription en littérature, le financement participatif est une pratique ancienne dans les mondes culturels. Mais le développement de plateformes numériques est une modalité supplémentaire de ce type de modèle qui conduit à une certaine adaptation des filières. Un documentariste explique, en entretien, que bénéficier d'un financement participatif entre aujourd'hui dans les critères du CNC pour le plan de préfinancement et dans la diffusion du film.

Le crowdfunding s'essouffle parfois, comme le précisent certains porteurs rencontrés, dans la mesure où ces derniers ne peuvent solliciter à plusieurs reprises leurs communautés. $\mathrm{Si}$ celles-ci contribuent lors de la première campagne, il est plus difficile de les mobiliser dans le temps : " II y a un épuisement des proches au bout de la troisième campagne ", confirme une réalisatrice. Un appel au financement participatif répond souvent à une logique d'expérience unique. Sur les pages de profil des porteurs de projets étudiés, il est en effet possible de comptabiliser le nombre de projets déposés sur Ulule depuis leur inscription sur la plateforme. Dès lors, presque $90 \%$ des porteurs de projets audiovisuels étudiés (contre $80 \%$ des porteurs de projets musicaux et $73 \%$ des projets éditoriaux) créent une seule campagne de financement participatif sur Ulule. Ceci s'explique peut-être par le temps long de réalisation d'un film (spécificité de la filière).

La mobilisation d'une communauté de donateurs dépend du type de projets. Quand il s'agit d'un projet militant ou de la 
Ulule, du courtage à la structuration des pratiques de création dans l'édition, la musique et l'audiovisuel

création d'un média, il semble plus aisé de faire appel plusieurs fois à la communauté, car elle se réunit autour d'une cause. Ainsi, selon les projets, le réseau sollicité diffère. Pour les enquêtés, il rassemble des proches, mais aussi une communauté d'intérêts (autour d'un porteur ou d'un projet). Dans ce dernier cas, les contributeurs sont construits sur le temps long autour d'une thématique, d'un sujet, d'un engagement sociétal et politique (par exemple, l'agriculture durable).

Il convient également de "différencier les projets qui ont besoin d'un coup de main, des projets où l'on vend un service (en prévente, en pré-abonnement) », comme l'indique un documentariste. Deux modes de collecte sont en effet proposés sur Ulule: "définir un objectif budgétaire, ou un nombre d'objets/articles à prévendre » (rubrique " $F A Q$ »). Le premier, qui correspond au modèle du don par contrepartie "en nature " (biens, services, rencontres liés au projet ou à ses porteurs) domine plus largement par rapport au second, celui de la souscription. En effet, seuls 23 des projets sur les 472 étudiés relèvent de la prévente (soit $4,87 \%$ des projets). Ce modèle est surtout présent dans le domaine de l'édition littéraire (15 projets sur les 23 en souscription relèvent de l'édition) où la pratique est ancrée. II s'avère néanmoins que le modèle de la contrepartie relève d'une forme de prévente déguisée en don.

D'un point de vue juridique, dès lors qu'un don donne lieu à une contrepartie (un bien, un service, etc.), il s'agit d'une vente. L'équipe d'Ulule l'indique pourtant clairement sur son site : « la plupart des transactions faites sur Ulule sont des ventes, même si les parties en présence n'en n'ont pas toujours conscience ". Si la notion de don apparaît très souvent dans les discours promotionnels et dans les représentations sociales liés au financement participatif, sa "réalité " apparaît donc peu pertinente du point de vue juridico-économique. La rhétorique du "don contre-don » fonctionne plutôt comme un attribut discursif et symbolique visant à conférer à Ulule une valeur d'usage liée à la culture du monde non marchand, stratégie particulièrement courante chez les acteurs du Web collaboratif. Ce positionnement idéologique, lié à ladite " économie 2.0 " (Rebillard, 2007) permet en effet de légitimer ce type de service et de distinguer la plateforme des acteurs plus traditionnels. 


\subsection{Une reconfiguration des compétences professionnelles}

Ulule participe d'un phénomène de transformations socioprofessionnelles au sein des industries culturelles: celle de l'artiste qui se mue en auteur-entrepreneur (Rouzé et Matthews, 2018), ou plus exactement qui revêt l'habit du "travailleur créatif type ». Comme l'indiquent Bouquillion, Miège et Mœglin (2013), ce dernier, "tel qu'il est présenté dans des rapports officiels européens sur les industries créatives, est un autoentrepreneur dans le cadre d'une économie libéralisée où la flexibilité au travail doit devenir la règle » (p. 67). Ces phénomènes apparaissent bien dans le cas du crowdfunding : "organisés sur un mode "horizontal" invitant à la collaboration, ils [les porteurs] doivent répondre à une polyvalence organisationnelle, logistique, budgétaire et se doivent de déployer des compétences diversifiées " (Matthews, Rouzé et Vachet, 2014, p. 318). Dans le cadre d'Ulule, les porteurs de projets (réalisateurs, musiciens, écrivains) endossent les rôles additionnels de producteurs ou d'éditeurs et, surtout, de marketeurs, de promoteurs ou de communicants.

Peu importe le projet, l'ensemble des porteurs est invité à publier des vidéos de présentation de celui-ci sur la page Ulule et donc, en creux, à détenir des compétences audiovisuelles (scénarisation, tournage et montage). Un paradoxe se dessine dans le fait que l'injonction à la création de contenus pour la page Ulule s'effectue sur des contenus culturels eux-mêmes en projets. Cette injonction à la formalisation de projet n'est pas initiée par le crowdfunding, mais est ancrée dans la culture professionnelle des créateurs, rompus depuis longtemps à l'exercice de convaincre des producteurs et des organismes subventionnaires souvent réticents à toute prise de risque artistique et financière. À ce titre, un réalisateur mentionne que "le fait de devoir courir derrière des financements et de défendre le projet a toujours fait partie du job de réalisateur ».

Ulule apporte toutefois une contribution à ces injonctions en conduisant, par l'intermédiaire de conseils, à la standardisation des formats de présentation de campagnes par l'imposition de titres, de sous-titres, en insistant sur la production d'une vidéo principale, explicative, esthétique et de courte durée (1 min $30 \mathrm{~s}$ à $2 \mathrm{~min}$ ), faite à partir d'images emblématiques et de contenus 
Ulule, du courtage à la structuration des pratiques de création dans l'édition, la musique et l'audiovisuel

à insérer (selon la règle des "CQQCOQP ${ }^{12}$ ") ou à éviter (" être quémandeur, contenir les mots soutiens ou aides"), ou encore sur l'intégration de liens vers ses comptes Facebook ou Twitter, son blogue ou ses pages personnelles. Ces injonctions implicites révèlent plusieurs enjeux. Elles renouvellent les barrières à l'entrée pour les créateurs et les producteurs d'une part et, d'autre part, à la visibilité sociale des projets. Ulule impose également des compétences relevant du storytelling : il s'agit qu' " un projet devienne un beau projet » en racontant « la genèse du projet » et en "construisant un univers textuel et visuel " autour de lui (cf. ulule.com). Les formes de standardisation de présentation apparaissent comme une conséquence de la nécessité de "faire parler » le projet à un ensemble disparate d'acteurs pouvant s'y impliquer: fans, publics, partenaires bancaires, chaînes de télévision.

Le travail des porteurs dépasse des tâches de réalisation pour assurer des missions de recommandation ou de promotion qui reviennent habituellement au distributeur ou au responsable marketing. Ulule remet un mode d'emploi aux porteurs où est expliqué comment créer une "entité " autour du projet, ou comment faire appel, d'abord, au "cercle proche ». Si des project managers sont prévus par l'équipe comme des adjuvants aux porteurs, tous les acteurs interrogés mentionnent ne jamais avoir été aidés pour promouvoir la campagne de collecte. Ces activités sont laissées aux porteurs ou aux contributeurs (donateurs).

Ces états de fait conduisent à des inégalités. Certains porteurs doivent tout apprendre, alors que d'autres ont déjà des connaissances dans le montage ou la promotion d'un projet. Si un producteur de musique, également chargé de communication dans une entreprise, a pu mener à bien sa collecte, c'est grâce à l'application de ses compétences professionnelles de communicant sur la campagne (élaboration d'un plan média). Être "entrepreneur de sa notoriété " (Beuscart, 2008) amène à développer des stratégies de visibilité du projet, d'animation de communautés ou de gestion de comptabilité, mais selon un calibrage imposé par Ulule.

Les porteurs sont ainsi conviés à alimenter la rubrique "news" de leur page afin de créer un lien avec les

${ }^{12}$ Comment ? Quoi ? Qui ? Combien ? Où ? Quand ? Pourquoi ? 
contributeurs. Ils réalisent des pages dédiées à leur projet sur les réseaux sociaux ou s'en font le relai (par des modalités de partage) sur d'autres. Pour un réalisateur rencontré, cela se traduit par un travail régulier et quotidien de communication (relance des contributeurs par les news, actualisation de la page Facebook, réponse aux commentaires) qu'il fait le soir après les moments de tournage. Pour d'autres, cela se concrétise par le recrutement, le temps de la campagne, d'une personne dédiée à ces activités de promotion, mais aussi d'envoi de colis, de calcul de frais de port des contreparties. La promotion sur les réseaux sociaux s'accompagne parfois d'évènements organisés en parallèle. Un documentariste explique en entretien : "On a organisé des soirées en librairie, pour la force de la rencontre en vrai. Les gens ont rempli des formulaires sur place, nous ont donné du cash ou des chèques. " L'ensemble de ces activités est chronophage et dépasse le temps de la campagne de crowdfunding. Comme le précise une réalisatrice interrogée: "Le mois avant la campagne, nous avons créé des évènements, les pages du projet sur les réseaux sociaux. » Enfin, les différents entretiens révèlent en réalité des formes de parasubordination (Cardon et Casilli, 2015) des porteurs de projet à la plateforme Ulule. Celles-ci se distinguent de la subordination classique du salariat, avec des micro-activités pas toujours imposées par contrat et soulignent des relations verticales entre la plateforme et les porteurs de projet.

Dès lors, Ulule n'est pas l'initiateur de bouleversements importants touchant les filières et ses activités socioprofessionnelles; la société contribue plutôt à dynamiser des mutations engagées de longue date. La plateforme s'insère ainsi dans des transformations en cours tout en y contribuant. Elle relève d'une activité socioéconomique d'intermédiation propre aux acteurs du Web (Bouquillion et Matthews, 2010), mais qui se caractérise ici spécifiquement par des mises en relation, parfois inédites et originales, entre acteurs variés ou par l'exercice d'un filtrage éditorial supplémentaire appliqué aux créateurs (la présélection des projets).

Ulule accompagne aussi l'avancée de logiques " collaboratives » dans les industries culturelles en renouvelant, notamment, les modèles de financement participatif qui existent de longue date. Cependant, si la plateforme octroie (avec ses 
Ulule, du courtage à la structuration des pratiques de création dans l'édition, la musique et l'audiovisuel

concurrentes et par les discours d'escorte), de par sa dimension "numérique ", une certaine "popularité " sociale au crowdfunding, sa prise en compte concrète par les acteurs institutionnels reste faible. Les porteurs ont également soulevé de très nombreuses difficultés liées à la mobilisation des collaborateurs-donateurs sur le temps long. Même le modèle du don désintéressé apparaît en réalité comme une logique de prévente. Ulule participe pleinement aux mutations socioprofessionnelles inhérentes aux créateurs et aux artistes, engagées depuis un certain temps et renouvelées avec le Web collaboratif, à savoir le développement de la «polyvalence organisationnelle » et notamment, ici, d'une compétence de « management de communautés».

\section{Des mobilisations différenciées}

Ulule relève-t-elle d'une forme d'homogénéisation des pratiques de création/production? Nous montrons ici qu'à l'inverse, celles-ci restent particulièrement différenciées et marquées par les caractéristiques socioéconomiques de la filière d'origine $\mathrm{du}$ projet, $\mathrm{du}$ statut socioprofessionnel $\mathrm{du}$ porteur et du type de plateforme mobilisée.

\subsection{Des variations d'une filière à l'autre}

Des différences fortes apparaissent dans les montants moyens demandés et récoltés de projets, et ce, en fonction de leur filière d'origine (Tableau 1). Parmi les projets en cours de financement, les projets musicaux collectent le plus d'argent (5 286 euros en moyenne), le nombre de contributeurs moyens y étant plus important: 262 contributeurs pour les projets musicaux contre 103 pour les projets éditoriaux et les projets audiovisuels. 
Tableau 1. Montant moyen demandé versus montant moyen récolté

Projets en cours de financement

\begin{tabular}{|l|l|l|l|}
\hline & Audiovisual & Musique & Edition \& Journal \\
\hline $\begin{array}{l}\text { Montant moyen } \\
\text { demandé }\end{array}$ & 4382 & 4696 & 4364 \\
$\begin{array}{l}\text { Montant moyen } \\
\text { récolté }\end{array}$ & 4539 & $\mathbf{5 2 8 6}$ & 4782 \\
\hline
\end{tabular}

Projets financés triés par montants récoltés

\begin{tabular}{|l|l|l|l|}
\hline & Audlovisuel & Musique & Edition \& Journal \\
\hline $\begin{array}{l}\text { Montant moyen } \\
\text { demandé }\end{array}$ & 22351 & 15956 & 36988 \\
$\begin{array}{l}\text { Montant moyen } \\
\text { récolté }\end{array}$ & 54912 & 24420 & $\mathbf{5 6 1 9 3}$ \\
\hline
\end{tabular}

Pour les projets triés par montant collecté, la tendance est quelque peu différente: les projets éditoriaux arrivent en tête (56 193 euros collectés en moyenne), suivis par les projets audiovisuels (54 912 euros) et, enfin, les projets musicaux (24 420 euros). Les projets ici soutenus sont engagés, militants ou fédérateurs pour venir en soutien à des médias en perte de vitesse (Rue89, Nice Matin) ou servir des causes (environnementales, sociales).

Ensuite, selon les filières, le taux d'échec des campagnes de financement participatif n'est pas le même. Ainsi, parmi les projets étudiés qui étaient en cours de financement au début de l'enquête, $20 \%$ des projets éditoriaux ne sont pas financés à l'issue de la campagne de crowdfunding, contre $13 \%$ dans la musique et $10,66 \%$ dans l'audiovisuel ${ }^{13}$.

En outre, des différences sont identifiables dans l'utilisation annoncée des contributions récoltées. Pour les projets audiovisuels (Tableau 2), qu'ils soient en cours de financement ou triés par montant collecté, l'analyse menée révèle que les porteurs sont essentiellement en recherche de financement pour la phase de réalisation-production du projet. Ils demandent des donations pour l'achat de matériels, le défraiement, les salaires, la régie ou les décors.

\footnotetext{
${ }^{13}$ Pour calculer le taux d'échec, nous avons mis à jour les données sur les montants récoltés pour chaque projet en cours de financement, dès la fin de leur campagne de crowdfunding sur Ulule.
} 
Ulule, du courtage à la structuration des pratiques de création dans l'édition, la musique et l'audiovisuel

Tableau 2. Répartition des contributions pour les projets audiovisuels

\begin{tabular}{|l|c|c|}
\hline & Projets en cours de financement & Projets financés \\
\hline $\begin{array}{l}\text { Réalisation- } \\
\text { production }\end{array}$ & $\mathbf{9 8}$ & $\mathbf{3 7}$ \\
\hline $\begin{array}{l}\text { Post-production } \\
\text { Distribution }\end{array}$ & 42 & 24 \\
\hline $\begin{array}{l}\text { Campagne de } \\
\text { crowdfunding }\end{array}$ & 23 & 10 \\
\hline
\end{tabular}

Concernant les projets musicaux en cours de financement (Tableau 3), la phase de distribution est privilégiée afin de soutenir financièrement les activités de graphisme, de pressage ou encore de promotion de l'album ou de l'EP. Pour les projets triés par montant collecté, il s'agit en revanche davantage de la phase de production, comprenant l'enregistrement (location de studios), les salaires, les défraiements ou l'achat de matériels. La postproduction (mixage, mastering), bien que présente, n'est pas la plus demandée.

Tableau 3. Répartition des contributions pour les projets musicaux

\begin{tabular}{|l|c|c|}
\hline & Projets en cours de financement & Projets finances \\
\hline Production & 65 & $\mathbf{3 7}$ \\
\hline Post-production & 57 & 17 \\
\hline Distribution & $\mathbf{8 1}$ & 31 \\
\hline $\begin{array}{l}\text { Campagne de } \\
\text { crowdfunding }\end{array}$ & 25 & 15 \\
\hline
\end{tabular}

Enfin, pour les projets éditoriaux en cours de financement (Tableau 4), les contributions viennent pour la plupart a priori financer la phase d'édition du livre: impression, maquette, rémunération et correction. Pour les projets triés par montant collecté, il est plus souvent question de la phase de conception : création de contenus, illustration, graphisme, achat de matériels ou défraiement. Dans ces projets se trouvent des projets de création de médias et non réellement de livres ; ils ne se situent donc pas à la même échelle. D'ailleurs, parmi ces projets, certains demandent de l'argent pour sortir de la faillite et permettre la sauvegarde d'emplois, le remboursement d'un 
prêt ou encore l'offre d'une modalité d'abonnement pour s'assurer la présence de lecteurs.

Tableau 4. Répartition des contributions pour les projets éditoriaux

\begin{tabular}{|l|c|c|}
\hline & Projets en cours de financement & Projets finances \\
\hline Conception & 34 & $\mathbf{2 8}$ \\
\hline Edition & $\mathbf{8 3}$ & 25 \\
\hline Distribution & 46 & 12 \\
\hline Faillite & 0 & 9 \\
\hline $\begin{array}{l}\text { Campagne de } \\
\text { crowdfunding }\end{array}$ & 42 & 2 \\
\hline
\end{tabular}

Enfin, soulignons que les régions d'origine des porteurs divergent selon le type de projets : $47 \%$ des porteurs de projets audiovisuels sont issus de la région Île-de-France, contre $33 \%$ pour les projets éditoriaux et $27 \%$ pour les projets musicaux. Cette répartition est assez symptomatique du découpage classique au sein des industries culturelles, notamment pour la filière cinématographique et audiovisuelle, la région Île-deFrance étant la première région de France dans ce domaine, suivie par la région Auvergne-Rhône-Alpes.

Ces variations reflètent les consistances propres des filières. Ulule ne bouscule pas réellement les procès à l'œuvre, mais emprunte à l'existant pour se situer dans les interstices de la chaîne de production de contenus culturels.

\subsection{Des utilisations divergentes selon les acteurs}

Entre individus " entrants », acteurs déjà "intégrés " dans les filières ou encore petites structures, les uns et les autres ont des regards (selon les entretiens menés) et des usages différenciés de la plateforme (d'après l'analyse des projets).

Pour les individus "entrants", Ulule est mobilisée comme un outil de préprofessionnalisation. Ainsi, plus d'un quart des projets audiovisuels en cours de financement $(27 \%)$ sont menés par des étudiants en audiovisuel, issus d'écoles comme I'ESEC, 3IS, I'ESRA ou CinéCréatis. Ils utilisent la plateforme comme un moyen de finaliser des projets de fin d'études au regard des descriptions des projets écrites sur Ulule. Certains y 
Ulule, du courtage à la structuration des pratiques de création dans l'édition, la musique et l'audiovisuel

voient un levier afin de nourrir un $\mathrm{CV}$ ou de construire un réseau professionnel. Ulule représente également un outil de commercialisation de projets culturels pour des " autodidactes » autoqualifiés. En effet, un réalisateur interrogé s'en sert comme un moyen de finaliser et de commercialiser des projets qu'il considère originaux sans passer par les acteurs classiques (qu'il ne connaît pas) ni par les étapes traditionnelles (qu'il ne maîtrise pas). C'est une façon de trouver une liberté éditoriale sur le sujet (la permaculture) et le format (un film à chapitres, à visée pédagogique), une communauté d'intérêts, une voie de diffusion (par une édition DVD soustitrée en plusieurs langues) et un moyen de visibilité, sans passer par les "rouages", qui seraient, selon lui, une contrainte au projet.

Pour les individus "intégrés " dans la filière, tels des réalisateurs plus "aguerris » (avec dix années d'expérience dans de la réalisation de films variés: courts métrages, documentaires, films d'entreprise), faire appel au crowdfunding s'inscrit dans des logiques de contournement, d'acceptation ou encore de communication autour du projet. Ulule incarne un moyen de contourner les temporalités longues des demandes de financement classique. Pour un réalisateur rencontré, le succès du crowdfunding traduit les difficultés et les échecs d'un système classique de production trop «frileux ». Le financement participatif est un "symptôme d'une défaillance " de ce système. Deux réalisateurs interrogés ajoutent que le crowdfunding est une manière de commencer sans attendre ni le tournage, ni le montage, ni la finalisation des dossiers de préfinancement, ni encore les réponses, souvent très longues, des acteurs du financement traditionnel. Dans les deux cas, la nature et le sujet du projet possible (un projet sur la maladie et un projet sur les inégalités sociales liées au contexte médiatique du moment) étaient particulièrement sensibles à la dimension temporelle et exigeaient que le tournage soit réalisé rapidement.

L'argument d'une campagne réussie est également mobilisé comme un moyen de faire accepter un projet à un producteur ou de le vendre à des diffuseurs. Un réalisateur interrogé explique que son producteur, ayant à l'origine des réserves sur un projet, avait besoin de "garanties visuelles » pour accepter de le financer. L'argent récolté par le crowdfunding a permis au 
réalisateur de lancer le tournage et de montrer des rushes au producteur, qui a fini par accepter. Une campagne réussie sur Ulule facilite aussi, pour les porteurs, l'obtention de subventions et de financement par les acteurs traditionnels de la production (aide $\mathrm{CNC}$ ) et de la diffusion (chaîne publique). L'utilisation d'Ulule se justifie enfin, pour les individus intégrés, comme un bon outil promotionnel sur le projet. Un documentariste précise, lors d'un entretien, que "le crowdfunding, c'est surtout une campagne de communication ».

Du point de vue des petites structures (des franges), le crowdfunding se dessine comme un tremplin pour impulser des projets. II facilite liberté, autonomie et indépendance, notamment pour des sociétés de production qui s'en saisissent et dont les revendications éditoriales sont fortes, telle Les films de la Falaise, qui porte " l'ambition de produire un cinéma "hors champ": indépendant, libre et engagé, pour résister aux conventions sociales et culturelles ». Ulule est aussi facilitateur en matière de visibilité et de reconnaissance. Un porteur énonce à ce sujet que "la campagne est un passage obligé pour qu'on parle de notre projet et que les producteurs nouveaux médias soutiennent symboliquement notre projet dans la liste des projets à venir intéressants ». Un réalisateur rencontré relate mettre le logo d'Ulule en avant et l'utiliser comme un "label " lors de projection en festival (en le plaçant sur l'affiche du film, la bande-annonce, le teaser). La présence de ce logo résulte du choix du réalisateur et de son producteur, non d'une obligation d'Ulule.

Ulule est majoritairement mobilisée par des créateurs (personnes physiques) plutôt que par des acteurs de la production ou de la diffusion (avec des variations selon les filières). Dans le cinéma, si des structures de production plus institutionnalisées (associations ou entreprises) y ont recours, ce sont plus souvent des petites structures liées aux créateurs, ayant par exemple leur propre entreprise, qui y font appel. Les acteurs de la production ou de la diffusion ont parfois des attitudes ambiguës avec le crowdfunding. Certains encouragent les créateurs à en faire sans les soutenir dans le déroulement de la campagne, et si la collecte réussit, ils l'instrumentalisent pour enrichir symboliquement et financièrement une demande de subvention. 
Ulule, du courtage à la structuration des pratiques de création dans l'édition, la musique et l'audiovisuel

\subsection{Des motivations circonstanciées selon les plateformes}

Les motivations des porteurs sous-tendant le recours au crowdfunding, comme les entretiens menés en témoignent, divergent selon le type de plateformes choisies. Celles intervenant dans le choix d'Ulule sont variées. Les porteurs insistent sur la dimension nationale de la plateforme. Son atout: " avoir des interlocuteurs français ", qui comprennent le projet. Mais ils soulignent aussi son ambition européenne : elle est alors perçue comme utile pour les projets pouvant être édités en Europe ou " compris " par des publics européens. La plateforme étant disponible en plusieurs langues (huit), elle offre une possibilité de traduction de son projet. Outre cette dimension de visibilité dans plusieurs pays, Ulule est également sélectionnée par les porteurs pour le design de la plateforme, modulable, permettant au porteur de mettre en valeur le projet et de personnaliser sa présentation. D'autres mettent en avant le côté "humain » de la plateforme, l'impression qu'il y a « quelqu'un derrière ». D'aucuns, enfin, l'utilisent tout simplement, car ils la connaissent déjà et préfèrent garder leurs repères.

Les motivations entrant en jeu dans l'utilisation d'autres plateformes sont notamment le fait de viser des publics anglophones (pour Kickstarter) ou de bénéficier d'un accompagnement spécialisé en cinéma, ou encore d'être exonéré de TVA sur l'appel de fonds (pour Touscoprod). Un éditeur de livres indique notamment que l'équipe d'Ulule, composée de «technophiles de la génération web», est coupée du monde de la vente. Selon lui, " les salariés d'Ulule sont sursollicités. Ils passent leur temps à refuser des projets pour ne garder que les plus susceptibles de réussir ". Pour lui, c'est le travail cumulé depuis 2000 qui lui a permis de réussir. Des porteurs critiquent la non-connaissance d'Ulule des modalités spécifiques du fonctionnement des filières des industries culturelles. D'aucuns critiquent encore la commission prise et l'assujettissement à la TVA pour les porteurs de projets. Pour contourner la commission, un porteur indique: "nous sommes allés jusqu'à la somme demandée et nous avons empoché directement le surplus!».

Finalement, la plateforme perpétue les caractéristiques socioéconomiques et socioprofessionnelles propres à chacune 
des industries culturelles, mais aussi à chacun des types d'acteurs et ses finalités. Dès lors, les besoins en financement ciblés sur certaines étapes clés des projets se raccordent aux logiques spécifiques et intrinsèques des filières. En d'autres termes, les demandes sont révélatrices des traits constitutifs de chacune d'entre elles et continuent de perdurer. Les projets et l'envergure des demandes en fonds sont à l'échelle du statut de leur porteur: travaux de fin d'études pour les « entrants » et documentaires pour les plus "expérimentés ». La plateforme révèle ainsi des formes de reproduction de l'existant.

\section{Conclusion}

Dans la continuité de travaux critiques récents relevant de l'économie politique de la communication (Bouquillion et Matthews, 2010; Matthews et Rouzé, 2018), nous avons montré qu'Ulule, plateforme de crowdfunding culturelle généraliste française, accompagne - à l'instar du Web collaboratif et en tant que plateforme d'intermédiation - un renforcement des formes d'industrialisation de la culture, notamment sur le plan des activités de création, qui s'en trouvent régulées à nouveaux frais. Quels sont les contours et les implications d'un tel constat? Ulule participe, selon le modèle du courtage, à un phénomène de structuration des pratiques de création conduisant à des formes d'élargissement du travail créatif (Cardon et Casilli, 2015 ; Matthews, Rouzé et Vachet, 2014). Les activités d'intermédiation de la plateforme s'opèrent plus précisément à deux niveaux au sein de la chaîne de coopération des industries culturelles.

À l'échelle des projets (niveau micro), la plateforme intervient de manière structurante dans la professionnalisation des projets par des conseils, des formatages ou d'autres standardisations en correspondance au marché (reproduisant une polarisation des contenus: de niches versus de masse). Ulule accompagne en définitive une (re)structuration des pratiques de création en orchestrant la hiérarchisation des rapports entre différents acteurs et en participant à la formalisation des contenus par des activités de sélection et d'accompagnement des productions. Les enjeux du crowdfunding pour les porteurs sont en effet divers.

II est d'abord question de formaliser toujours plus les projets. 
Ulule, du courtage à la structuration des pratiques de création dans l'édition, la musique et l'audiovisuel

Cette formalisation se note dans les injonctions données aux porteurs à devoir prouver le potentiel de réussite d'une campagne, intégrer leur projet dans une présentation standardisée et le vendre à des contributeurs. II s'agit ensuite, dans le cadre de la campagne de financement participatif, de développer le projet avec des acteurs hors filière culturelle, assez hétérogènes (acteurs financiers, acteurs de l'alimentation, etc.). Ces relations se construisent dans les activités de production (commandites, partenariats), de communication et de promotion, ou encore d'aide à la collecte des contributions. Enfin, les projets sont laissés à leur porteur, que ce soit pendant la collecte (l'investissement minimal des acteurs traditionnels en témoigne) ou après la collecte (Ulule se désiste alors totalement). Ulule invite ainsi à des recompositions des jeux et des compétences professionnelles. Elle conduit les auteurs-réalisateurs à être davantage valorisés comme travailleurs créatifs et à s'affirmer comme tels.

À l'échelle industrielle (niveau méso), Ulule joue un rôle de structuration dans la mise en relation des acteurs (rôle de vivier, de facilitateur de mise en contact, de laboratoire grandeur nature). Ce rôle dévolu à la plateforme ne conduit pas forcément à des formes de réorganisation des filières, mais contribue à un renforcement de tendances industrielles qui se font plus pressantes : sophistication de l'intermédiation, gestion du risque d'incertitude, financiarisation croissante, facilitation de l'entrée d'acteurs exogènes dans les filières culturelles, structuration de manière indirecte de parties sousindustrialisées de la filière, à l'échelle des sous-filières (telle la $\mathrm{BD}$ ) ou au croisement de filières (tels les clips vidéo).

En définitive, les marques d'Ulule sur le fonctionnement socioéconomique et professionnel des industries culturelles se « moulent » dans l'existant. Loin de bouleverser des économies structurées depuis longtemps, elle se qualifie plutôt comme un acteur supplémentaire qui, sans remplacer ses prédécesseurs, s'y additionne. II conviendrait dès lors d'actualiser ce constat en identifiant plus clairement et systématiquement, sur la durée, les modalités par lesquelles le recours au crowdfunding peut se systématiser ou se pérenniser en suivant d'une part des porteurs sur une trajectoire professionnelle plus longue et, d'autre part, des projets finalisés et mis sur le marché (dépassant dès lors le stade du projet et leur conversion en 
bien d'expérience). II s'agirait de changer d'échelle d'analyse et de construire une démarche monographique par professionnel ou par projet.

Au-delà du cas d'Ulule et sur un plan macro-économique, il est aussi possible d'émettre quelques réserves sur l'envergure et les enjeux socioéconomiques du crowdfunding culturel sur Internet. Si le marché français dans sa globalité reste dynamique et en hausse $(+44 \%$ de fonds collectés entre 2016 et 2017 , dont $+20 \%$ pour le modèle du don ${ }^{14}$ ), force est de constater que le «secteur culturel ${ }^{15}$ » du financement participatif (45 millions de fonds collectés en 2017) reste assez modeste au regard du "secteur économique ${ }^{16}$ " (261 millions en 2017). Ces chiffres suggèrent aussi que le crowdfunding n'est plébiscité que par des acteurs minoritaires au sein des industries culturelles (producteurs ou distributeurs dans le cinéma, labels de musique). Pour le cas du cinéma, une étude prospective du CNC de 2015 montre que le crowdfunding reste largement plébiscité pour les petits projets : courts métrages et films d'animation ${ }^{17}$.

II est aussi révélateur de constater que les plateformes culturelles spécialisées sur un type de contenu culturel ont soit disparu, soit ont été rachetées (People for cinema, par Ulule), soit se sont diversifiées (MyMajorCompany), soit restent marginales (Movies Angels). Elles ne parviennent pas à trouver le "succès» de leurs concurrentes "généralistes", telles Ulule ou KissKissBankBank, qui sont leader en France. Mais celles-ci semblent désormais accorder moins d'importance aux projets liés à des contenus culturels qu'à ceux fondés sur des dimensions sociales, solidaires ou "créatives ». II reste ainsi nécessaire de suivre l'évolution du positionnement stratégique

\footnotetext{
${ }^{14}$ Baromètre du crowdfunding 2017 (KPMG et association financement participatif France) : http://financeparticipative.org/barometres-crowdfunding/barometre-ducrowdfunding-2017/

${ }^{15}$ Cette catégorie est reprise de l'association Financement participatif France, qui y regroupe: édition, journalisme, publications, arts plastiques, spectacle vivant, audiovisuel, musique, patrimoine.

${ }^{16}$ Cette catégorie, issue de la même source, rassemble : immobilier, environnement et énergies renouvelables, industrie, agriculture, commerces et services, technologie et numérique, santé et recherche scientifique.

17 Danard, B. (2015, octobre). Le financement participatif : un nouveau modèle de financement?. Étude prospective présentée lors de la table-ronde "Tous producteurs ! Le cinéma, l'audiovisuel et le financement participatif », CNC et School of public Affairs de Sciences Po.
} 
Ulule, du courtage à la structuration des pratiques de création dans l'édition, la musique et l'audiovisuel

et éditorial de ces plateformes dont l'implication dans les industries culturelles, si elle est minoritaire, n'en reste pas moins incertaine.

\section{Références}

Benghozi, P.-J. (dir.) (2013). Entreprises culturelles et internet : contenus numériques et modèles d'affaires. Paris, France: Questions de culture.

Bénistant, A. et Marty, E. (2016, 8 novembre). Les médias et le crowdfunding: une analyse des représentations médiatiques. The Conversation. Repéré à https://theconversation.com/les-medias-et-le-crowdfundingune-analyse-des-representations-mediatiques-68242

Beuscart, J. (2008). Sociabilité en ligne, notoriété virtuelle et carrière artistique: les usages de MySpace par les musiciens autoproduits. Réseaux, (152), 139-168.

Bouquillion, P. (2008). Les industries de la culture et de la communication. Les stratégies du capitalisme. Grenoble, France : Presses universitaires de Grenoble.

Bouquillion, P. et Matthews, J. (2010). Le web collaboratif. Mutations des industries de la culture et de la communication. Grenoble, France : Presses universitaires de Grenoble.

Bouquillion, P., Miège, B. et Moeglin, P. (2013). L'industrialisation des biens symboliques. Les industries créatives en regard des industries culturelles. Grenoble, France : Presses universitaires de Grenoble.

Bubendorff, S. (2014). Le financement participatif, dispositif de désintermédiation du processus de création ? Expériences et discours des artistes. Les enjeux de l'information et de la communication, (15/2a), 21-30. Repéré à http://lesenjeux.ugrenoble3.fr/2014-supplementA/02-Bubendorff/index.html

Bullich V. (2015). Régulation des pratiques amateurs et accompagnement de la professionnalisation : la stratégie de YouTube dans la course aux contenus exclusifs. Les enjeux de l'information et de la communication, (16/3B), 27-42. Repéré à http://lesenjeux.u-grenoble3.fr/2015supplementB/02-Bullich/index.html 
Cardon, D. et Cassilli, A. (2015). Qu'est ce que le digital labor?. Paris, France : Ina Éditions.

Chantepie, P. et Le Diberder, A. (2005). Révolution numérique et industries culturelles. Paris, France : La Découverte.

Creton, L. et Kitsopanidou, K. 2016 (dir.), Crowdfunding, industries culturelles et démarche participative. De nouveaux financements pour la création. Paris, France : Peter Lang.

Dupuy-Salle, M. (2016) Le financement participatif : quelle innovation pour la production cinématographique et audiovisuelle?. Dans L. Creton et K. Kitsopanidou (dir.), Crowdfunding, industries culturelles et démarche participative: de nouveaux financements pour la création? (p. 159-173). Paris : Éditions Peter Lang

Guibert, G., Rebillard, F. et Rochelandet, F. (2016). Médias, culture et numérique. Approches socio-économiques. Paris, France : Armand Colin.

Huet A. et al. (1978). Capitalisme et industries culturelles. Grenoble, France : Presses universitaires de Grenoble.

Matthews, T. J., Rouzé, V. et Vachet, J. (2014). La culture par les foules? Le crowdfunding et le crowdsourcing en question. Paris, France : MkF Éditions.

Matthews, T. J. (2015). Passé, présent et potentiel des plateformes collaboratives. Réflexions sur la production culturelle et les dispositifs d'intermédiation numérique. Les enjeux de l'information et de la communication, (16), 57-71. Repéré à http://lesenjeux.u-grenoble3.fr/2015/04Matthews/index.html

Miège, B. (2017), Les industries culturelles et créatives face à l'ordre de l'information et de la communication. Grenoble, France : Presses universitaires de Grenoble.

Mœglin, P. (2007). Des modèles socio-économiques en mutation. Dans P. Bouquillion et Y. Combes (dir.), Les industries de la culture et de la communication en mutation (p. 151-162). Paris, France : L'Harmattan.

Mœglin, P. (2012). Une théorie pour penser les industries culturelles et informationnelles?. Revue française des sciences de l'information et de la communication, (1). doi: 10.4000/rfsic. 130 
Ulule, du courtage à la structuration des pratiques de création dans l'édition, la musique et l'audiovisuel

Onnée, S. et Renault, S. (2013). Le financement participatif : atouts, risques et conditions de succès. Gestion, 38(3), 5465.

Perticoz, L. (2012). Les industries culturelles en mutation : des modèles en question. Revue française des sciences de l'information et de la communication, (1). doi: 10.4000/rfsic. 112

Rallet, A. et Rochelandet, F. (2016). Financement participatif et valorisation des biens culturels: vers plus de diversité culturelle?. Dans L. Creton et K. Kitsopanidou (dir.), Crowdfunding, industries culturelles et démarche participative. De nouveaux financements pour la création (p. 57-87). Paris, France : Peter Lang.

Rouzé, V. et Matthews, T. J. (2018). Les plateformes de crowdfunding culturel : entre figures de l'artiste entrepreneur et entrepreneurs polymorphes. Les enjeux de l'information et de la communication, (19), 35-50. Repéré à https://lesenjeux.univ-grenoble-alpes.fr/2018/03-RouzeMatthews/

Rebillard, F. (2007). Le Web 2.0 en perspective. Une analyse socio-économique de l'Internet. Paris, France : L'Harmattan.

Uricchio, W. (2014). Repenser le documentaire social. Dans L. Allard, L. Creton et R. Odin (dir.), Téléphonie mobile et création (p. 62-79). Paris, France : Armand Colin. 